\title{
MEDICALIZAÇÃO E TDAH: DISCUSSÕES NOS PROGRAMAS DE PÓS- GRADUAÇÃO EM EDUCAÇÃO E EM PSICOLOGIA DO MATO GROSSO DO SUL
}

\author{
MEDICALIZATION AND TDAH: DISCUSSIONS IN GRADUATE PROGRAMS IN \\ EDUCATION AND PSYCHOLOGY OF THE MATO GROSSO DO SUL
}

\author{
Mayara Karolina Alvarenga Recaldes Gomes Coutinho ${ }^{1}$ \\ Doracina Aparecida de Castro Araujo 2
}

\begin{abstract}
Resumo
Estudo bibliográfico, de abordagem qualitativa com o objetivo de compreender a abordagem dos temas Medicalização e Transtorno do Déficit de Atenção e Hiperatividade (TDAH) na produção científica dos campos da Educação e Psicologia no Estado de Mato Grosso do Sul. Empregou-se a metodologia do estado do conhecimento, com um recorte temporal e espacial em levantamentos realizados nas teses e dissertações defendidas nos Programas de PósGraduação em Educação e em Psicologia cujo resumo contivesse os descritores medicalização ou TDAH. Os dados permitiram afirmar que o volume de pesquisas em Educação e em Psicologia em Mato Grosso do Sul é significativo, considerando a recente implantação da maioria dos programas, entretanto, as pesquisas sobre as temáticas em estudo ainda são incipientes necessitando de mais pesquisas que abordem a medicalização na Educação.
\end{abstract}

Palavras-chave: Medicalização. TDAH. Educação. Psicologia.

\begin{abstract}
Bibliographical study, a qualitative approach in order to understand the approach of the themes Medicalization and attention deficit hyperactivity disorder (TDAH) in the scientific production of the fields of Education and psychology in the State of Mato Grosso do Sul. The methodology employed in the State of knowledge, with a temporal and spatial in surveys carried out in theses and dissertations defended in graduate programs in education and in psychology whose summary contained medicalization descriptors or TDAH. The data allowed the volume of research in education and Psychology in Mato Grosso do Sul is significant, considering the recent implementation of most programs, however, the research on the themes under study are still incipient requiring more research that address the medicalization in education.
\end{abstract}

Keywords: Medicalization. TDAH. Education. Psychology.

\footnotetext{
${ }^{1}$ Mestranda do Programa de Pós-Graduação em Educação da Universidade Estadual de Mato Grosso do Sul (UEMS), Unidade Universitária de Paranaíba. Psicóloga da Universidade Estadual de Mato Grosso do Sul, Campus de Paranaíba. E-mail: mayarakargcoutinho@gmail.com.

${ }^{2}$ Doutora em Educação pela Universidade Estadual de Campinas (UNICAMP). Pós-doutorado em Educação Escolar pela Universidade Estadual Paulista "Júlio de Mesquita Filho" (UNESP/Araraquara). Docente do Programa de Pós-Graduação em Educação e do curso de Pedagogia da UEMS, Unidade Universitária de Paranaíba. Coordenadora do Centro de Ensino, Pesquisa e Extensão em Educação (CEPEED) da UEMS. Líder do Grupo de Estudos e Pesquisas em Práxis Educacional (GEPPE). E-mail: doracina@gmail.com.
} 


\section{Introdução}

Este trabalho se insere na interface entre as áreas de Educação e Psicologia, ao propor a análise da abordagem do tema da medicalização implicada no Transtorno do Déficit de Atenção e Hiperatividade (TDAH). Trata-se de um estudo tipo estado do conhecimento que objetivou compreender a abordagem dos temas Medicalização e TDAH nas teses e dissertações defendidas em Programas de Pós-Graduação em Educação e em Psicologia do Estado de Mato Grosso do Sul, a partir de uma abordagem qualitativa.

Collares e Moysés (2011) contextualizam o processo de medicalização da educação dentro da biologização da vida, que consiste na transformação de questões sociais em questões biológicas e, portanto, individuais, ancorada numa lógica positivista de ciência que importa para as ciências humanas o modelo do método científico das ciências biológicas. Nesse sentido, compreendemos a medicalização como o “[...] processo de transformar questões não médicas, eminentemente de origem social e política, em questões médicas, isto é, tentar buscar no campo médico as causas e soluções para problemas dessa natureza”. (COLLARES; MOYSÉS, 1994, p. 25).

Ao passo que o TDAH configura-se atualmente como uma queixa escolar demandante de avaliação psicológica, os estudos sobre a medicalização da vida e da escola trazem o questionamento da queixa escolar e de alguns processos de avaliação psicológica. Neste sentido, a partir da queixa escolar configurada numa queixa de desatenção e agitação motora características do TDAH, a escola procura as respostas na saúde, em busca de um diagnóstico que confirme a suposta doença que impede o aprender, atribuindo ao fracasso escolar uma etiologia orgânica.

A metodologia adotada foi a pesquisa bibliográfica do tipo estado do conhecimento. Primeiramente, foi efetuada a quantificação e identificação dos dados bibliográficos: mapeamento da produção segundo período, locais e áreas de produção e, em seguida, realizou-se o inventário da produção por meio da análise qualitativa a partir das seguintes categorias de análise: (1) definição dos conceitos medicalização e TDAH, (2) metodologia empregada e (3) Referencial teórico. 


\section{O ensino superior no Brasil e em Mato Grosso do Sul}

A Universidade é entendida como instituição necessária à constituição de uma nação e cursar uma graduação reveste-se de grande relevância social, especialmente nas regiões mais pobres do Brasil. Neste sentido, para Severino (2008) a universidade é a instituição responsável pela formação dos profissionais e "preparação dos quadros administrativos e das lideranças culturais e sociais do país, sendo visto como poderoso instrumento de ascensão social” (SEVERINO, 2008, p. 74). O mesmo autor situa, historicamente, a implantação sistemática das Universidades no Brasil a partir da terceira década do século XX. A seguir, são apresentadas brevemente algumas peculiaridades da implantação do ensino superior no Mato Grosso do Sul.

Como é sabido, o Mato Grosso do Sul se constituiu a partir da divisão do Estado de Mato Grosso em 1977, por sua vez, a educação escolar primária foi introduzida no Estado de Mato Grosso pelos religiosos salesianos em 1894 com o objetivo inicial de evangelização indígena, posteriormente houve o investimento na educação de jovens e adultos e a expansão para a educação formal em várias regiões do Estado (BITTAR, 2002 apud BARBOSA, 2012). Os salesianos alcançaram a região de Campo Grande, no sul do Mato Grosso em 1912, com a inauguração da Escola de São José, conforme Bittar (2002) citada por Barbosa (2012), os salesianos ampliaram sua área de atuação desde a educação primária ao ginásio, adentrando ao Ensino Superior com a oferta dos cursos de Letras e Pedagogia em Campo Grande, em 1961 e a criação da Faculdade Dom Aquino de Filosofia, Ciências e Letras, lançando as bases para a primeira universidade salesiana do país, a Universidade Católica Dom Bosco (UCDB), aprovada em 1993. A UCDB está localizada na cidade de Campo Grande e oferta presentemente 34 cursos de graduação, seis cursos de mestrado e quatro cursos de doutorado. Para alcançar os objetivos desta pesquisa, nossa área de interesse centrou-se nos cursos de mestrado e doutorado em Educação e em Psicologia ${ }^{3}$.

Com o desmembramento do estado de Mato Grosso ocorreu a implementação do Mato Grosso do Sul em 1979, assim os campi da Universidade Estadual de Mato Grosso presentes no território delimitado para o Estado do Mato Grosso do Sul foram federalizados, constituindo a Universidade Federal de Mato Grosso do Sul (UFMS). A UFMS oferece atualmente 138 cursos de ensino superior e tecnólogo, nas modalidades presencial e à

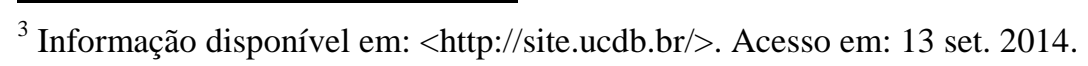


distância, distribuídos em dois centros, seis faculdades, três institutos e dez campi ${ }^{4}$. Quanto à pós-graduação stricto sensu, em nível de mestrado, a UFMS oferece 35 cursos. Dentro de nossa área de estudo, encontramos um curso de mestrado em Psicologia em Campo Grande, e dois cursos de mestrado em Educação, um em Campo Grande e outro no campus de Corumbá. Em nível de doutorado, a UFMS dispõe de 14 cursos, sendo um em Educação em Campo Grande 5 .

A história da Universidade Estadual de Mato Grosso do Sul (UEMS) é marcada pelo “enfrentamento entre o poder político e o intelectual" (SILVA-FILHO, 2008, p. 14). Tal assertiva encontra respaldo quando observamos no sítio da universidade, que se transcorreu um período de tempo significativo entre sua criação pela Constituição Estadual de 1979 e a autorização pelo Ministério da Educação em 1997, passando por uma ratificação na Constituição Estadual de 1989 e sendo instituída pela Lei $n^{\circ}$ 1461, de 20 de dezembro de 1993. ${ }^{6}$ A UEMS está sediada em Dourados e ramificada em 15 unidades, oferece 61 cursos de graduação. A pós-graduação stricto sensu oferta sete cursos de mestrado acadêmico, cinco de mestrado profissional e um curso de doutorado ${ }^{7}$. Em nossa pesquisa, nos detivemos no curso de mestrado acadêmico em Educação da Unidade Universitária de Paranaíba e no curso de mestrado profissional em Educação de Campo Grande.

A Universidade Federal da Grande Dourados (UFGD) foi criada em 2006 com a alocação dos cursos de graduação e pós-graduação do Centro Pedagógico de Dourados, que até então, estava ligado à UFMS. Atualmente, a UFGD possui 11 faculdades, 33 cursos de graduação, 18 cursos de mestrado, e oito cursos de doutorado. Seguindo nosso interesse de pesquisa, nos focamos nos cursos de mestrado e doutorado em Educação.

Apesar de todas as dificuldades decorrentes da cultura oligárquica, o ensino superior público expandiu-se e o Estado conta hoje com três instituições públicas, duas federais (UFMS, UFGD) e uma estadual (UEMS) além das instituições de ensino superior privadas (UCDB, UNIDERP). Os próximos tópicos contemplarão o histórico dos programas de pósgraduação em Educação e em Psicologia no Mato Grosso do Sul, assim como apresentarão os resultados da coleta de dados.

\footnotetext{
${ }^{4}$ Informação disponível em: <http://www.sien.ufms.br/>. Acesso em: 13 set. 2014.

${ }^{5}$ Informação disponível em: <https://sistemas.ufms.br/sigpos/portal/>. Acesso em: 13 set. 2014.

${ }^{6}$ Informação disponível em: <http://www.uems.br/internet/historia.php>. Acesso em: 13 set. 2014.

${ }^{7}$ Informação disponível em: < http://www.portal.uems.br/>. Acesso em: 13 set. 2014.
} 


\subsection{Os programas de pós-graduação em Educação do Mato Grosso do Sul}

Observamos que os programas de pós-graduação em nível de mestrado ainda são em número pequeno (seis), porém distribuem-se pelas regiões Leste (UEMS-Paranaíba), Central (UFMS, UCDB, UEMS-Campo Grande), Sul (UFGD-Dourados) e Oeste (UFMS-Corumbá), mas tem maior concentração em Campo Grande, onde funcionam três cursos. Quanto aos programas em nível de doutorado, notamos a presença de três cursos, dois na região Central, em Campo Grande (UFMS e UCDB) e um na região sul (UFGD), este dado é compreensível dada a recente implantação dos programas e mesmo o histórico do Ensino Superior no Estado.

\subsubsection{Universidade Católica Dom Bosco}

O Programa de Pós-Graduação em Educação (PPGEdu) da $\mathrm{UCDB}^{8}$ tem como objetivo geral formar docentes pesquisadores qualificados para atividades de pesquisa, ensino, extensão e assessoria na área da Educação, com formação que possibilite a compreensão das questões educacionais a partir do conhecimento dos fundamentos históricos, sociológicos filosóficos e epistemológicos da Educação. Para tanto, desde 1994 a UCDB fornece o mestrado em Educação, o qual obteve o reconhecimento do curso pela Coordenação de Aperfeiçoamento de Pessoal de Nível Superior (CAPES) em 2002. O doutorado em Educação da UCDB foi aprovado em 2009 e implantado em 2010.

As linhas de pesquisa constituintes do PPGEdu da UCDB são três, a saber: diversidade cultural e educação indígena; políticas educacionais, gestão escolar e formação docente; e práticas pedagógicas e suas relações com a formação docente. A primeira linha de pesquisa integra estudos sobre a diversidade cultural e as relações entre educação, cultura, multiculturalismo e interculturalidade referentes às realidades das comunidades indígenas e afrodescendentes e à educação popular e aos movimentos sociais. A segunda linha de pesquisa, políticas educacionais, gestão escolar e formação docente, contempla estudos que analisam os processos macroestruturais que fundamentam as políticas públicas sociais, especialmente a interferência dos organismos internacionais na formulação e concretização das políticas públicas no âmbito escolar. A terceira linha de pesquisa do PPGEdu da UCDB, apresenta estudos com temáticas mais abrangentes acerca das relações entre a natureza e a

\footnotetext{
${ }^{8}$ Informações disponíveis em: <http://site.ucdb.br/cursos/4/mestrado-e-doutorado/32/doutorado-emeducacao/2618/>. Acesso em: 25 jun. 2014.
} 
constituição do saber escolar e seu tratamento didático-pedagógico, bem como sobre o professor e a formação docente inicial e continuada.

Das três linhas de pesquisa do PPGEdu da UCDB, observamos que somente a última apresenta em sua descrição uma aproximação com a Psicologia ao apontar que considera a dimensão psicopedagógica envolvida no processo de ensino e aprendizagem. Deduzimos ser esta a razão das temáticas medicalização e TDAH não ocuparem a agenda das pesquisas, posto que estas implicam a necessária interface entre os campos da Psicologia e da Educação. A seguir apresentamos as tabelas com os dados resultantes da busca pelos descritores "medicalização" e "TDAH" nos resumos das dissertações e teses defendidas no PPGEdu da UCDB.

Tabela 1. Dissertações PPGEdu UCDB

\begin{tabular}{c|c|c|c}
\hline Ano & N. trabalhos & Medicalização & TDAH \\
\hline 1996 & 11 & 0 & 0 \\
\hline 1997 & 07 & 0 & 0 \\
\hline 1998 & 24 & 0 & 0 \\
\hline 1999 & 19 & 0 & 0 \\
\hline 2000 & 19 & 0 & 0 \\
\hline 2001 & 0 & 0 & 0 \\
\hline 2002 & 14 & 0 & 0 \\
\hline 2003 & 18 & 0 & 0 \\
\hline 2004 & 03 & 0 & 0 \\
\hline 2005 & 19 & 0 & 0 \\
\hline 2007 & 22 & 0 & 0 \\
\hline 2008 & 18 & 0 & 0 \\
\hline 2009 & 16 & 0 & 0 \\
\hline 2010 & 26 & 0 & 0 \\
\hline 2011 & 20 & 0 & 0 \\
\hline 2012 & 11 & 0 & 0 \\
\hline TOTAL & 11 & 0 & 0 \\
\hline
\end{tabular}

Fonte: Site da UCDB. Disponível em:

<http://www3.ucdb.br/mestrados/index.php?c_mestrado=>. Acesso em: 10 ago. 2014.

Ao longo dos 18 anos do curso de mestrado em Educação na UCDB, observamos uma média aproximada de 15 trabalhos defendidos por ano, sendo os picos de produção em 2009, 1998 e 2006 com 26, 24 e 22 trabalhos defendidos respectivamente, por outro lado, observamos o ano de 2001, com nenhuma produção, o ano de 2004 com três dissertações defendidas, e os anos de 2011 e 2012 com 11 trabalhos defendidos em cada ano; concluímos, assim, que não há uma uniformidade na distribuição dos trabalhos por ano letivo. Quanto ao nosso objeto de estudo, os dados revelam que em nenhum dos resumos das 258 dissertações defendidas no PPGEdu da UCDB foram localizados os descritores medicalização e TDAH, demonstrando que as temáticas em estudo nesta monografia não se fizeram presentes nos 
trabalhos defendidos. Dado semelhante foi encontrado na pesquisa nos resumos das teses, como pode ser observado na tabela 2.

Tabela 2. Teses PPGEdu UCDB

\begin{tabular}{c|c|c|c}
\hline Ano & N. trabalhos & Medicalização & TDAH \\
\hline 2013 & 02 & 0 & 0 \\
\hline 2014 & 02 & 0 & 0 \\
\hline TOTAL & $\mathbf{0 4}$ & $\mathbf{0}$ & $\mathbf{0}$ \\
\hline
\end{tabular}

Fonte: Site da UCDB. Disponível em: <http://site.ucdb.br/cursos/4/mestrado-edoutorado/32/doutorado-em-educacao/2618/teses-defendidas/2626/> Acesso em: 10 ago. 2014.

Conforme os dados disponíveis, o curso de doutorado em Educação da UCDB teve início em 2010, assim, as primeiras teses defendidas datam de 2013. Encontramos somente quatro teses defendidas até o momento, sendo que em nenhuma delas figuram os temas medicalização e TDAH.

\subsubsection{Universidade Federal de Mato Grosso do Sul}

O programa de pós-graduação em Educação da UFMS Campo Grande ${ }^{9}$, que agrega o mestrado e o doutorado em Educação, se originou do Curso de Mestrado em Educação implantado em 1988 mediante convênio com a Faculdade de Educação da Universidade Estadual de Campinas (FE-UNICAMP). Inicialmente o curso de mestrado tinha um projeto curricular amplo que permitiu a inclusão de professores de diversos departamentos e campi da UFMS. O convênio com a FE-UNICAMP findou-se em 1991 e em 1996 o curso de mestrado em Educação passou por um processo de avaliação da CAPES que resultou na organização em Linhas de Pesquisa e na constituição do PPGEdu.

Atualmente, o PPGEdu da UFMS em Campo Grande tem como objetivo principal iniciar e consolidar a formação de pesquisadores em Educação e estimular a produção científica no campo educacional. O PPGEdu está estruturado em cinco linhas de pesquisa: Educação e trabalho; ensino de ciências e matemática; história, políticas e Educação; escola, cultura e disciplinas escolares; e Educação, Psicologia e prática docente. Abaixo, a tabela 3 apresenta os dados levantados na pesquisa pelos descritores medicalização e TDAH nos resumos das dissertações do PPGEdu da UFMS em Campo Grande.

\footnotetext{
${ }^{9}$ Informações disponíveis em: <http://www.ppgedu.ufms.br/>. Acesso em: 10 ago. 2014.
} 
Tabela 3. Dissertações PPGEdu UFMS Campo Grande.

\begin{tabular}{c|c|c|c}
\hline Ano & N. produções & Medicalização & TDAH \\
\hline 1991 a 1999 & 99 & 0 & 0 \\
\hline 2000 & 06 & 0 & 0 \\
\hline 2001 & 14 & 0 & 0 \\
\hline 2002 & 21 & 0 & 0 \\
\hline 2003 & 22 & $\mathbf{0 1}$ & 0 \\
\hline 2004 & 20 & 0 & 0 \\
\hline 2005 & 31 & 0 & 0 \\
\hline 2006 & 35 & 0 & 0 \\
\hline 2007 & 23 & 0 & 0 \\
\hline 2008 & 17 & 0 & 0 \\
\hline 2009 & 22 & 0 & 0 \\
\hline 2010 & 18 & 0 & 0 \\
\hline TOTAL & $\mathbf{3 2 8}$ & $\mathbf{0 1}$ & $\mathbf{0}$ \\
\hline
\end{tabular}

Fonte: Site da UFMS. Disponível em: <http://www.ppgedu.ufms.br>. Acesso em: 10 ago. 2014.

O primeiro aspecto a ser salientado é que o site do PPGEdu da UFMS de Campo Grande, contrariando as disposições da CAPES, não disponibiliza o acesso aos resumos e às demais informações das dissertações defendidas depois do ano de 2010. Assim, embora não tenhamos delimitado o espaço temporal para as buscas, enquanto nos programas da UDCB e da UEMS encontramos dados mais recentes, o PPGEdu da UFMS limitou as buscas ao ano de 2010 .

Quanto ao nosso objeto de estudo, foi localizada uma dissertação defendida no ano de 2003 que contém em seu resumo a palavra medicalização. Trata-se do trabalho da psicóloga Ivonete Bitencourt Antunes Bittelbrunn, intitulado O silêncio da escola pública: um estudo sobre os programas de atendimento aos alunos com indicadores de superdotação no estado de Mato Grosso do Sul e orientado pela professora doutora Alexandra Ayache Anache.

Em sua dissertação, Bittelbrunn (2003) objetivou compreender o discurso oficial do Estado de Mato Grosso do Sul para a educação dos alunos que apresentavam indicadores de superdotação, matriculados na Rede Estadual de Ensino, por meio da análise dos documentos produzidos pelo Estado sobre o atendimento deste público durante o período de 1979, ano da implementação do Mato Grosso do Sul, até 2001, ano do início da pesquisa. O referencial teórico adotado pela autora foi a abordagem histórico-cultural e o método empregado foi a pesquisa qualitativa.

Quanto à metodologia, a autora procedeu a um estudo exploratório para explicitar a realidade educativa do Mato Grosso do Sul no que tange ao aluno superdotado, buscando na Secretaria Estadual de Educação os projetos voltados ao atendimento a estes alunos. Em 
seguida a autora realizou uma análise documental a partir da análise de conteúdo elencando as categorias de análise conceito de superdotado, conceito de inteligência, processo de identificação de alunos superdotados, propostas pedagógicas e profissionais envolvidos.

Em relação aos resultados, Bittelbrunn (2003) encontrou semelhanças na estruturação dos programas de atendimento a alunos superdotados no Estado e na federação, apontou para a tensão entre continuidade e descontinuidade das iniciativas governamentais para o atendimento destes alunos, exemplo disso é que os três projetos analisados iniciam e terminam dentro de determinada gestão estadual, não tendo continuidade na gestão seguinte. Outro ponto levantado pela autora é que os programas estaduais estão alinhados à proposta federal do Ministério da Educação e Cultura, baseadas no pressuposto da "Educação para todos”. Bittelbrunn (2003) assinalou, em seu trabalho, a distância entre o ideal da Educação para Todos e a realidade encontrada em sua pesquisa.

No tocante à análise documental, foram avaliados três projetos, dos anos de 1987, 1996 e 2001. Bittelbrunn (2003) conclui que a descontinuidade dos programas devido às mudanças de gestão, apontadas acima, permitem considerar que o atendimento efetivo dos alunos superdotados não é realizado, devido à burocratização dos serviços educacionais e escolares. Para a autora, é urgente o momento de surgir e consolidar políticas públicas consistentes que possibilitem à escola se constituir como organizadora de práticas pedagógicas que considerem os interesses dos alunos, os ritmos de aprendizagem e o desenvolvimento psicológico considerando a sistematização do conhecimento e a vinculação entre escola e sociedade.

No trabalho de Bittelbrunn (2003) a medicalização é abordada na análise da categoria "profissionais envolvidos". Dois dos três projetos analisados referem-se aos profissionais especializados, em sua maioria, na área da saúde (psicólogos, fisioterapeutas, assistentes, sociais e pedagogos) como responsáveis pelo atendimento dos alunos com indicadores de superdotação. Para a autora, tal fenômeno, evidencia a correspondência entre Educação Especial e Atendimento Especializado, "reafirmando uma postura medicalizante da Educação Especial” (BITTELBRUNN, 2003, p. 88). O risco da medicalização da Educação Especial é a oferta de atendimentos especializados em detrimento daqueles que pertencem à função da escola - ensinar, transmitir conhecimento e possibilitar a produção de novos conhecimentos. Para a autora, quando os atendimentos especializados se fizerem necessários, devem ser realizados pelo setor da Saúde, mas o que se observa é a demanda constante por profissionais 
especializados dentro da escola, o que pode suprimir a verdadeira função dela e sua atividade principal - o ensino - que deve ser ofertado pelo professor.

Em nível de doutorado, as primeiras defesas do PPGEdu da UFMS de Campo Grande ocorreram no ano de 2008. Assim como ocorre com as dissertações, o site do PPGEdu da UFMS Campo Grande não disponibiliza as informações das teses defendidas após 2010. Como pode ser observado na tabela 4 abaixo, entre os anos de 2008 e 2010 foram defendidas 23 teses, sendo uma tese defendida em 2008 e 11 em 2009 e 2010, respectivamente.

\section{Tabela 4. Teses PPGEdu UFMS Campo Grande.}

\begin{tabular}{c|c|c|c}
\hline Ano & N. produções & Medicalização & TDAH \\
\hline 2008 & 01 & 0 & 0 \\
\hline 2009 & 11 & 0 & 01 \\
\hline 2010 & 11 & 0 & 0 \\
\hline TOTAL & $\mathbf{2 3}$ & $\mathbf{0}$ & $\mathbf{0 1}$ \\
\hline
\end{tabular}

Fonte: Site da UFMS. Disponível em: 〈http://www.ppgedu.ufms.br〉 Acesso em: 10 ago. 2014.

Na busca pelo descritor medicalização não encontramos resultados, porém na busca pelo descritor TDAH encontramos uma tese defendida em 2009. Trata-se do trabalho da psicóloga Ieda Maria Munhos Benedetti, orientado pela professora doutora Alexandra Ayach Anache, intitulado "Transtorno de déficit de atenção e hiperatividade: os (des)caminhos da construção de um conceito". Infelizmente não encontramos no site do PPGEdu da UFMS Campo Grande a publicação do trabalho completo, diante disso, consultamos o acervo da biblioteca central da UFMS em Campo Grande, por intermédio da bibliotecária do campus de Paranaíba e foi informado que não se encontram registros da cópia física da tese. Deste modo, a descrição a seguir foi realizada somente com as informações contidas no resumo da tese, disponível no site do PPPGEdu da UFMS Campo Grande.

Benedetti (2009) em sua tese buscou analisar o processo de construção do conceito TDAH, elucidando seus pontos críticos, os sujeitos diagnosticados, a escola e a família envolvida, os tratamentos e a bibliografia existente sobre o TDAH. O método empregado no estudo foi a pesquisa qualitativa, o resumo deixa claro somente que houve "casos entrevistados", porém não relata o número de casos e demais procedimentos metodológicos. Os resultados da pesquisa de Benedetti (2009) estruturam-se em quatro momentos, o primeiro traz a fundamentação metodológica da pesquisa e o segundo faz uma análise da escola e de suas recentes transformações, da família e da sociedade de consumo a partir de uma vertente crítica ao capitalismo contemporâneo. A terceira parte do estudo traz uma ampla revisão da 
literatura sobre o TDAH a partir do viés positivista, calcado na experimentação, quantificação e objetivação do objeto de estudo, posição hegemônica da abordagem dos fenômenos alocados na sigla TDAH. Por fim a autora propõe um diálogo com diversos autores de posicionamento crítico a esta visão hegemônica, especialmente a abordagem Psicodinâmica e Histórico-Cultural.

Quanto aos resultados, Benedetti (2009) afirma que as manifestações subjetivas contemporâneas têm sido apropriadas pela ciência positivista que, respondendo à lógica capitalista, nomeia, define, afere e propõe estratégias de cura para os novos diagnósticos, que se tornam elemento de consumo. Neste sentido, a autora conceitua o TDAH como um megadiagnóstico condensador de outros diagnósticos e de várias questões numa única sigla. As consequências deste processo, segundo a autora, são a simplificação das questões subjetivas, a culpabilização do sujeito diagnosticado e a desresponsabilização da família e da escola, que são eximidas da necessidade de repensar suas práticas e vinculações. Por fim, a autora conclui que o TDAH pode realmente existir a partir da ciência positivista, mas é um conceito superdimensionado que esconde erros "cometidos nos campos da ética, da má formação profissional, da banalização da medicamentação, do excesso de consumo de medicamentos, da superficialidade das análises e das leviandades ideológicas" (BENEDETTI, 2009). Embora a palavra medicalização não apareça no resumo da tese, as discussões aproximam-se da conceituação apresentada no primeiro capítulo desta monografia.

A UFMS conta ainda com mais um programa de pós-graduação em Educação localizado na cidade de Corumbá, no denominado Campus do Pantanal (CPAN). O curso teve sua proposta aprovada pela CAPES no segundo semestre de 2008 e o ingresso de sua primeira turma no início de 2009. Conforme as informações disponíveis no site do programa ${ }^{10}$, o curso de mestrado culmina o trabalho de 15 anos de pesquisa e extensão dos professores do CPAN nas temáticas infância, adolescência e exclusão social, realizado junto ao Centro de Referência e Estudos da Infância e Adolescência (CREIA). O curso de mestrado do PPGEdu da UFMS/CPAN tem como objetivo a formação de pesquisadores com sólido conhecimento na área da Educação, especialmente em Educação Social, área de concentração do programa; o preparo dos ingressantes para o magistério no Ensino Superior, enquanto professorpesquisador; a formação de pesquisadores, docentes e gestores para o desenvolvimento de

\footnotetext{
${ }^{10}$ Disponível em: <http://ppgecpan.sites.ufms.br/> . Acesso em: 20 ago. 2014.
} 
atividades nos diferentes níveis e modalidades do sistema educacional; e a produção científica sobre Educação e Educação Social.

O PPGEdu da UFMS/CPAN tem como área de concentração a Educação Social, com a proposta de construir interfaces entre a educação formal e não formal por meio da produção científica acerca dos processos educativos considerando o movimento da sociedade e suas contradições e desenvolver pesquisas focalizando a garantia dos direitos à educação, saúde, assistência e trabalho da população de crianças, adolescentes e suas famílias. Nesta área de concentração, o programa busca ainda desenvolver pesquisas sobre a implantação, implementação e avaliação de políticas públicas voltadas à infância e à adolescência; subsidiar a elaboração de políticas públicas, especialmente àquelas voltadas à população infanto-juventil; e produzir conhecimento sobre a formação de educadores em situação de educação formal e não formal.

Para alcançar estas propostas, o PPGEdu da UFMS em Corumbá estrutura-se em duas linhas de pesquisa: políticas, práticas institucionais e exclusão/inclusão social e formação de educadores e diversidade. A primeira linha de pesquisa abarca estudos sobre os diferentes cenários socioeconômicos e culturais da sociedade contemporânea e as relações com a construção dos direitos humanos, especialmente, daqueles relativos à infância e à adolescência. A linha de pesquisa Formação de educadores e diversidade tem como foco a formação de professores pesquisadores com saberes e práticas atentas às necessidades urgentes e emergentes da sociedade contemporânea e aprofundar o conhecimento acerca da formação e trabalho do educador nas situações de educação formal e não formal. Esta linha abrange o estudo das relações pedagógicas necessárias à articulação entre educação formal e não formal salientando o desenvolvimento humano e os processos de construção do conhecimento e o acesso ao conhecimento em contextos de diversidade/identidade. A tabela a seguir sistematiza a produção de dissertações do PPGEdu UFMS/CPAN segundo nosso objeto de estudo.

Tabela 5. Dissertações PPGEdu UFMS/CPAN.

\begin{tabular}{c|c|c|c}
\hline Ano & N. produções & Medicalização & TDAH \\
\hline 2011 & 08 & 0 & 0 \\
\hline 2012 & 06 & 0 & 0 \\
\hline TOTAL & $\mathbf{1 4}$ & $\mathbf{0}$ & $\mathbf{0}$ \\
\hline
\end{tabular}

Fonte: Site da UFMS/CPAN.

Disponível em: <http://ppgecpan.sites.ufms.br/category/dissertacoes-defendidas/2012/> Acesso em: 20 ago. 2014. 
Como pode ser visto, são 14 as dissertações defendidas, dado que a primeira turma do mestrado ingressou em 2009, as defesas ocorreram em 2011 e 2012, sendo oito defesas em 2011 e seis defesas em 2012. Tal como acontece com o PPGEdu de Campo Grande, o site do PPGEdu do CPAN não se encontra atualizado, assim, não apresenta as dissertações de 2013 e 2014, um atraso menor em relação ao programa de Campo Grande, mas ainda existente. Quanto ao nosso objeto de estudo, os temas medicalização e TDAH não foram contemplados nas dissertações defendidas no PPGEdu, uma vez que a busca pelos descritores não retornou resultados.

\subsubsection{Universidade Estadual de Mato Grosso do Sul}

A Universidade Estadual de Mato Grosso do Sul apresenta dois programas de pósgraduação em Educação, sendo um mestrado acadêmico na Unidade Universitária de Paranaíba e um mestrado profissional na unidade de Campo Grande, assim, contempla distintas regiões do Estado. O PPGEdu de Paranaíba teve sua aprovação em 2010 pelo Conselho de Ensino, Pesquisa e Extensão (CEPE) e em 2011 pela CAPES. Sua primeira turma ingressante foi em agosto de 2011. O PPGEdu da UEMS Paranaíba tem como área de concentração Educação, Linguagem e Sociedade, sendo que seu primeiro projeto pedagógico contemplava duas linhas de pesquisa: Linguagem, literatura educação e sociedade e Teorias e práticas educacionais. Já o projeto pedagógico atual, aprovado em 2013 e iniciado em 2014, apresenta três linhas de pesquisa, sendo a primeira Currículo, formação docente e diversidade, a segunda, História, sociedade e educação e a terceira, Linguagem, educação e cultura (UEMS, 2013).

O atual projeto pedagógico do PPGEdu da UEMS Paranaíba elenca cinco objetivos para o programa:

I - formar docentes e pesquisadores que atendam aos desafios da educação, para o exercício da profissão na Educação Básica e no Ensino Superior e para o desenvolvimento de pesquisas que concorram para o avanço do conhecimento, com competência científica e responsabilidade social;

II - propiciar a construção de conhecimentos de forma a responder aos desafios sociais, teóricos e metodológicos no campo da educação, linguagem e sociedade, proporcionando aos docentes e discentes subsídios teórico-metodológicos que possam contribuir para a melhoria na qualidade da educação, em seus diversos níveis e modalidades;

III - incentivar o desenvolvimento e fortalecimento das linhas de pesquisa do Programa, com vistas à realização de pesquisas nas esferas local, regional, nacional e internacional; 
IV - incentivar a divulgação do conhecimento e as produções realizadas pelos docentes e discentes do Programa por meio de eventos científicos e publicações; V - articular ações do Programa com os cursos da Universidade, em especial, a graduação, com observação às orientações estabelecidas pelos órgãos competentes da UEMS. (UEMS, 2013, p. 28).

Em relação à área de concentração, Educação, linguagem e sociedade, encontramos a reunião de docentes pesquisadores, com formação em Educação ou áreas afins, que investigam teorias e práticas educacionais em diferentes contextos e locais, tendo o sujeito como referência e fundamentados teórica e metodologicamente na abordagem sócio-históricocultural. Quanto às linhas de pesquisa alocadas nesta área de concentração notamos que a primeira, Currículo, formação docente e diversidade contempla estudos com

[...] temáticas voltadas para os aspectos cognitivos, psicológicos, políticos, sociais, culturais e históricos da educação; a formação docente inicial e continuada na Educação Básica e no Ensino Superior em diferentes áreas do conhecimento; o currículo; as questões sobre diversidade e inclusão de gênero, raça, etnia e educação especial em espaços escolares e não escolares; as violências que afetam o processo de ensino e aprendizagem: violência escolar, doméstica, questões ligadas a disciplina, indisciplina, cultura e poder na escola; educação prisional e violência, entre outros temas. (UEMS, 2013, p. 27).

Já a segunda linha de pesquisa, História, Sociedade e Educação,

[...] enfoca estudos teórico-metodológicos atinentes à história e historiografia da educação, contemplando as relações entre história, sociedade e educação, em investigações sobre formação e profissão docente; instituições escolares e nãoescolares; disciplinas escolares e currículo; saberes, prescrições, práticas e processos educativos; produção, circulação e apropriação de ideias e modelos educativos; impressos pedagógicos e manuais de ensino; escolarização da infância; educação indígena; universalização da escola pública; correntes educacionais contemporâneas. (UEMS, 2013, p. 27)

Por fim, a linha de pesquisa Linguagem, educação e cultura contempla três eixos, o primeiro, estudo da linguagem, abrange temas como alfabetização, letramento e multiletramentos. O segundo eixo refere-se ao estudo dos gêneros discursivos e textuais. $\mathrm{O}$ terceiro eixo, por sua vez, busca estudar temas relacionados a textos literários, literatura infantil e infanto-juvenil. A articulação com a Educação se dá por meio do estudo de aspectos sociais, culturais, históricos, políticos e filosóficos, e das "questões relativas ao processo ensino/aprendizagem; formação docente; memórias e narrativas de alunos e professores; questões identitárias; cultura e cultura escolar". (UEMS, 2013, p. 28).

A tabela 6 apresenta o resultado das buscas pelos descritores medicalização e TDAH nos resumos das dissertações defendidas no programa: 
Tabela 6. Dissertações PPGEdu UEMS, Unidade Universitária de Paranaíba.

\begin{tabular}{c|c|c|c}
\hline Ano & N. produções & Medicalização & TDAH \\
\hline 2012 & 01 & 0 & 0 \\
\hline 2013 & 14 & 0 & 0 \\
\hline 2014 & 20 & 0 & 0 \\
\hline TOTAL & $\mathbf{3 5}$ & $\mathbf{0}$ & $\mathbf{0}$ \\
\hline
\end{tabular}

Fonte: Site da UEMS. Disponível em:

<http://www.uems.br/pgedu/index.php?p=Disserta\%E7\%F5es> Acesso em: 25 ago. 2014

Conforme observamos, o PPGEdu já conta com 35 defesas nos seus três anos de funcionamento, sendo uma defesa em 2012, catorze dissertações em 2013 e vinte dissertações em 2014. Entretanto, não encontramos os descritores pesquisados nos resumos das dissertações, concluímos, assim, que as pesquisas não contemplaram a temática em estudo neste trabalho.

A UEMS mantém ainda um programa de mestrado profissional na Unidade Universitária de Campo Grande, aprovado em maio de 2012, o programa tem a área de concentração Formação de Educadores e duas linhas de pesquisa, a saber: Organização do Trabalho Didático e Formação de Professores e Diversidade. O mestrado profissional em Educação da UEMS ainda não apresenta dissertações defendidas.

\subsubsection{Universidade Federal da Grande Dourados}

O programa de pós-graduação da UFGD $^{11}$ iniciou suas atividades em 2008, tem como área de concentração História, políticas e gestão da educação e apresenta as seguintes linhas de pesquisas: história da educação, memória e sociedade; políticas e gestão da educação; e educação e diversidade. As linhas de atuação prioritárias do PPGEdu da UFGD são educação municipal, educação superior no contexto do Mercado Comum do Sul (MERCOSUL) e a relação entre educação e diversidades. Dentre os objetivos do programa destacamos a formação inicial de pesquisadores na área da educação a partir do fornecimento de condições teóricas, metodológicas e epistemológicas; fomentar a pesquisa educacional institucional, enfocando a realidade municipal, regional e/ou nacional; oferecer a formação inicial para a docência no ensino superior na área da educação e/ou quadros para a administração

\footnotetext{
${ }^{11}$ Informações disponíveis em: <http://www.ufgd.edu.br/faed/mestrado-educacao/historico> Acesso em: 25 ago. 2014.
} 
universitária; e a realização de pesquisas voltadas ao subsídio da concepção, implantação e avaliação das políticas públicas na área da educação, com ênfase nos âmbitos local e regional.

Quanto ao nosso objeto de estudo, o resultado das buscas pelos descritores medicalização e TDAH podem ser observados na tabela 7 .

Tabela 7. Dissertações PPGEdu UFGD.

\begin{tabular}{c|c|c|c}
\hline Ano & N. produções & Medicalização & TDAH \\
\hline 2010 & 13 & 0 & 0 \\
\hline 2011 & 17 & 0 & 0 \\
\hline 2012 & 18 & 0 & 0 \\
\hline TOTAL & $\mathbf{4 8}$ & $\mathbf{0}$ & $\mathbf{0}$ \\
\hline
\end{tabular}

Fonte: Site da UFGD. Disponível em:

<http://www.uems.br/pgedu/index.php?p=Disserta\%E7\%F5es> Acesso em: 25 ago. 2014.

A primeira observação a realizar refere-se ao fato do site do programa não disponibilizar as informações das dissertações defendidas em 2013. Conforme observamos, há um número crescente de dissertações defendidas no PPGEdu da UFGD, saindo de um total de 13 dissertações em 2010, para 17 em 2011 e 18 em 2012. Entretanto, em nenhuma das dissertações encontramos os descritores que orientam esta pesquisa. Faz importante registrar que o PPGEdu da UFGD também oferece pós-graduação stricto sensu em Educação em nível de doutorado, porém, o site do programa não fornece os dados do histórico do programa, nem teses defendidas.

\subsection{Os programas de pós-graduação em Psicologia do Mato Grosso do Sul}

O Estado de Mato Grosso do Sul conta com dois programas de pós-graduação stricto sensu em Psicologia (PPGPsi), sendo um em instituição de ensino superior privada e outro em instituição de ensino superior pública.

Ao contrário dos programas de pós-graduação em Educação, em que notamos uma disseminação pelas diferentes regiões do Estado, os programas de pós-graduação em Psicologia concentram-se na capital, Campo Grande. No Estado, temos um mestrado oferecido pela UFMS e um mestrado e um doutorado ofertados pela UCDB, pioneira também na área da pós-graduação em Educação. A seguir, apresentamos os históricos dos PPGPsi e os resultados da pesquisa. 


\subsubsection{Universidade Católica Dom Bosco}

O programa de pós-graduação em Psicologia (PPGPsi) da UCDB teve suas atividades em nível de mestrado iniciadas em 1997, sendo, assim, o curso de mestrado em Psicologia mais antigo do Estado. Conforme as informações obtidas no site ${ }^{12}$, o PPGPsi está alicerçado em pesquisas e experiências de diversas áreas do conhecimento, que contemplam aspectos culturais, sociais, de saúde, educação e históricos, da realidade local. Uma das prioridades do PPGPsi é a ampla integração dos trabalhos institucionais, com o Núcleo de Estudos Multidisciplinares de Psicologia (NEMPSI) e com os profissionais, pesquisadores e instituições brasileiras e internacionais na área de Psicologia ou em áreas afins, prioridade ampliada com a implantação do doutorado em 2013. Deste modo, assim como o PPGEdu da UCDB, O PPGPsi tem importante inserção nacional e internacional, com a participação de pesquisadores doutores, mestres e de convidados de outras instituições ligadas à pesquisa no Brasil e no exterior. A área de concentração do PPGPsi é Psicologia da Saúde e duas as linhas de pesquisa: práticas em saúde e qualidade de vida e políticas públicas, cultura e produções sociais. A primeira linha procura subsidiar investigações voltadas para a qualidade de vida, articulando saúde e adoecimento como eixo constitutivo das práticas em saúde. Esta linha de pesquisa sustenta-se nas políticas contemporâneas no que tange aos aspectos de proteção, promoção e recuperação em saúde e suas pesquisas, a partir de distintas modalidades de avaliação de indivíduos e coletivos, enfatizando a articulação entre as dimensões psicológicas, biológicas e sociais constitutivas da qualidade de vida.

A segunda linha de pesquisa do PPGPsi da UCDB, políticas públicas, cultura e produções sociais, engloba estudos que focalizam a subjetividade a partir da articulação entre saúde, política, cultura e produções sociais. Os estudos desta linha de pesquisa investigam temáticas relativas à articulação entre saúde, cultura e produções sociais a partir de uma perspectiva interdisciplinar e intersetorial.

Em relação ao nosso objeto de estudo, no mestrado em Psicologia da UCDB encontramos o maior número de produções com os descritores pesquisados, conforme observamos na tabela 8 a seguir.

\footnotetext{
${ }^{12}$ Informação disponível em: <http://site.ucdb.br/cursos/4/mestrado-e-doutorado/32/mestrado-empsicologia/607/sobre-o-programa/639/> Acesso em: 30 ago. 2014.
} 
Tabela 8. Dissertações PPGPsi UCDB

\begin{tabular}{c|c|c|c}
\hline Ano & n. trabalhos & Medicalização & TDAH \\
\hline 2003 & 36 & 0 & 0 \\
\hline 2004 & 06 & 0 & 0 \\
\hline 2005 & 16 & 0 & 0 \\
\hline 2006 & 14 & 0 & 0 \\
\hline 2007 & 29 & 01 & 0 \\
\hline 2008 & 12 & 0 & 0 \\
\hline 2009 & 20 & 0 & 0 \\
\hline 2010 & 12 & 0 & 0 \\
\hline 2011 & 11 & 0 & 01 \\
\hline 2012 & 15 & 01 & 0 \\
\hline 2013 & 10 & 0 & 0 \\
\hline TOTAL & 181 & 02 & 01 \\
\hline
\end{tabular}

Fonte: Site da UCDB. Disponível em:

<http://www3.ucdb.br/mestrados/index.php?c_mestrado=10> Acesso em: 30 ago. 2014.

No período de uma década, encontramos o expressivo número de 181 trabalhos defendidos, com uma média de 16 trabalhos por ano, todos com as respectivas informações disponíveis, exceto 11 trabalhos do ano de 2013, que apresentavam título e autor, mas não tinham o resumo publicado. A partir das buscas nos resumos, foram localizados dois trabalhos em medicalização (2007 e 2012) e um trabalho em TDAH (2011). Como ainda não há teses defendidas no PPGPsi da UCDB, passamos à apresentação em ordem temática dos trabalhos encontrados.

Ao realizarmos as buscas pelo descritor medicalização encontramos a dissertação de Helenrose da Silva Pedroso Coelho, que possui graduação em Ciências Sociais (1982), Direito (1992) e Psicologia (2004) ${ }^{13}$. Com o título A circulação dos sentidos de promoção da saúde e de prevenção de doenças na mídia impressa, a pesquisa foi orientada pela professora doutora Ângela Elisabeth Lapa Coêlho e defendida no ano de 2007.

Coelho (2007) procurou analisar os sentidos de promoção da saúde e prevenção de doenças presentes no caderno Folhaequilíbrio do jornal Folha de São Paulo durante o ano de 2005. Os objetivos específicos da pesquisa foram identificar e analisar os dados sociodemográficos dos profissionais e pessoas entrevistadas que subsidiaram as matérias, identificar as temáticas das chamadas de capa, analisar os discursos adotados no enfoque dado à promoção da saúde e prevenção de doenças, e analisar os repertórios dados às expressões

\footnotetext{
${ }^{13}$ Informação disponível em: <http://buscatextual.cnpq.br/buscatextual/visualizacv.do?id=K4139174H2> Acesso em: 13 set. 2014.
} 
promoção da saúde e prevenção de doenças nos conteúdos das matérias. Com isso a autora procurou enfocar como as formas de interagir e de se comunicar orientados pela mídia impressa atuam no meio social possibilitando a interpretação e reinterpretação dos sentidos de prevenção de doenças e promoção de saúde, bem como tornou possível à autora verificar o modelo adotado no enfoque dado ao processo saúde-doença.

A pesquisa de Coelho (2007) é classificada pela autora como pesquisa documental. O método empregado foi a análise descritiva e exploratória, utilizando a "[...] técnica de análise do conteúdo com a prática de análise do discurso" (COELHO, 2007, p. 107). Deste modo, a autora realizou uma análise quantitativa do material de pesquisa - todas as edições do Caderno Folhaequilíbrio publicadas em 2005 - apontando o perfil do leitor e dos entrevistados, temas recorrentes e enfoque da saúde. Também foi realizada uma análise qualitativa dos dados que buscou investigar os sentidos atribuídos às expressões promoção da saúde e prevenção de doenças emitidas por especialistas e pessoas entrevistadas para as matérias assim como das imagens colocadas nas matérias. As categorias elencadas pela análise do conteúdo das matérias foram: promoção da saúde e prevenção de doenças; mercantilização da saúde/consumismo; saúde como alimentação; culpabilização das pessoas; desqualificação do discurso não-científico e terapias alternativas; visão ampliada de saúde; discurso crítico; e banalização. (COELHO, 2007, p. 108).

A autora não examina a questão da medicalização da educação, mas a medicalização da vida atrelada à produção de sentidos sobre promoção da saúde e prevenção de doenças. Podemos afirmar, então, que a medicalização da vida é especialmente abordada na análise dos resultados. Para tanto, Coelho (2007) recorreu ao referencial teórico do materialismo histórico-dialético, proposto por Marx e Engels e, ao adentrar a questão da medicalização, apoiou-se nas proposições de Foucault em relação ao biopoder.

Como resultados de pesquisa, destacamos a medicalização vista como instrumento de biopoder, pois expande para outros campos as explicações biológicas forjadas no âmbito médico. A medicalização da vida também se reflete no conceito de saúde traduzido como ausência de doenças, e da saúde como um processo fragmentado passível de ser alcançado por meio de estilos de vida e formas adequadas de consumo. A autora conclui que inexiste no periódico analisado uma visão crítica sobre o adoecer e as relações socioculturais envolvidas no processo saúde-doença. Outro dado importante, demonstrado por Coelho (2007) é o modo como as relações de consumo contaminam o conteúdo e a visão de saúde e doença veiculados pelo caderno em análise, banalizando os diferentes estados de saúde, uma vez que para todos 
há uma explicação biológica ou baseada na bioquímica cerebral; há ainda a presença de modismos e moral julgadora, especialmente quanto às dicas para a vida saudável, reforçando estereótipos.

A segunda dissertação a mencionar o processo de medicalização foi a do teólogo e psicólogo Mario Balduino de Oliveira Junior ${ }^{14}$, Representações sociais de saúde e doença em professores de uma universidade pública do Mato Grosso do Sul, orientado pelo professor doutor Márcio Luis Costa, defendida no PPGPsi da UCDB em 2012. A pesquisa foi realizada junto a professores de uma instituição de ensino superior pública, localizada em Campo Grande, Mato Grosso do Sul, com o objetivo de levantar e circunscrever as representações sociais de saúde e doença deste grupo de sujeitos pesquisado.

O embasamento teórico desta pesquisa-intervenção qualitativa foi a Teoria das Representações sociais e a metodologia empregada, entrevistas semiestruturadas, com os instrumentos questionário sócio demográfico e roteiro de entrevista, ambos construídos pelo autor. Assim, as falas dos entrevistados foram analisadas levando em consideração não somente os aspectos concretos e lógicos, mas também os aspectos subjetivos simbólicos a partir da tematização das falas. Deste modo, o autor elencou duas temáticas que se constituíram representações sociais do grupo pesquisado: representação social de saúde e representação social de doença.

A questão da medicalização, tal como ocorre na primeira dissertação apresentada, não está vinculada à educação e formação de diagnósticos. Oliveira-Junior (2012) analisa a medicalização na representação social de saúde, apontando que este tema surge na fala dos entrevistados como uma ação a ser evitada, mesmo diante da dor física e/ou mal-estar subjetivo, sendo assim um significante de saúde. Desta forma, concluímos que o autor associa a medicalização ao uso de medicamentos alopáticos. Para a discussão pretendida por nosso estudo, a medicalização da vida e da educação vai além do uso de medicamentos, embora em muitos casos, o uso de destes esteja em jogo na medicalização, este processo não se restringe a isto. Antes, a medicalização, a partir do referencial teórico elencado no primeiro capítulo, trata-se de um processo mais amplo que envolve a explicação biológica de questões da vida cotidiana que extrapolam o campo de atuação médica, discussão esta que não foi encontrada no trabalho de Oliveira-Junior (2012).

\footnotetext{
${ }^{14}$ Informação disponível em: <http://buscatextual.cnpq.br/buscatextual/visualizacv.do?id=K4518462H5> Acesso em: 13 set. 2014
} 
No PPGPsi da UCDB, no ano de 2011, encontramos ainda a dissertação As influências da equitação terapêutica no desenvolvimento de crianças e adolescentes com transtorno de déficit de atenção/hiperatividade. De autoria da psicóloga ${ }^{15}$ Camila Spengler Escobar e orientado pela professora doutora Heloisa Bruna Grubits Freire, este trabalho foi encontrado nas buscas dirigidas pelo descritor TDAH.

Escobar (2011) parte do pressuposto de que a equitação terapêutica é um método que propicia benefícios biopsicossociais para o indivíduo, como melhoras referentes à socialização, aspectos afetivos e emocionais e que estimula aspectos relacionados à atenção, capacidade de concentração e desempenho escolar, importantes para o desenvolvimento de crianças e adolescentes com TDAH. No trabalho em estudo, o TDAH é definido como um transtorno mental diagnosticado na presença de sintomas de desatenção e hiperatividade, que interferem negativamente no ambiente familiar e escolar, conforme o DSM-IV. A partir destes pressupostos, a autora objetivou, com seu trabalho de pesquisa, verificar possíveis mudanças de comportamento em ambiente familiar, escolar e terapêutico, depois de oito meses de intervenção com Equoterapia/Equitação Terapêutica.

Em sua pesquisa, Escobar (2011) utilizou o método Hipotético-Dedutivo com delineamento Quase-Experimental de Série Temporal, com avaliações pré e pós-intervenção de sete sujeitos diagnosticados com TDAH, encaminhados por médico neurologista, e com idade compreendida entre sete e quatorze anos, sendo que apenas três faziam uso de medicação para controlar os sintomas do transtorno. Quanto aos procedimentos, inicialmente foram realizadas entrevistas com os pais e com os professores, orientadas por questionários. Posteriormente, a atenção e a capacidade de concentração dos sujeitos da pesquisa foram avaliados por meio do teste psicológico D2- Atenção Concentrada, também foram utilizados os testes de desempenho escolar, com os sujeitos e a Escala de TDAH - versão para professores, para análise dos comportamentos característicos do TDAH nos participantes em ambiente escolar. Os testes foram reaplicados ao final dos atendimentos aos sujeitos para avaliação dos resultados. Nos atendimentos foram utilizadas técnicas de Equitação pela autora.

Quanto aos resultados, a autora aponta que o processo terapêutico da equoterapia trouxe benefícios aos sujeitos participantes da pesquisa, os quais apresentaram redução significativa nos sintomas do TDAH e melhoras relacionadas à atenção, concentração, sono,

\footnotetext{
${ }^{15}$ Informação disponível em: <http://buscatextual.cnpq.br/buscatextual/visualizacv.do?id=K4735073A5> Acesso em: 13 set. 2014.
} 
disciplina, responsabilidade, agressividade, impulsividade, irritabilidade, dentre outras. Diante disso, Escobar (2011) conclui que a Equoterapia/Equitação Terapêutica é eficaz para o tratamento do TDAH, pois, além dos benefícios elencados, também promove o processo de aprendizagem. Ademais, a autora destaca que "o aprendizado é mais estável e consistente em crianças, pré-adolescentes e adolescentes com TDAH que não fazem uso de medicação para o transtorno; ou seja, estes, quando medicados estão propensos a regredir mais no mesmo período de tempo". (ESCOBAR, 2011, p. 142).

Observamos na dissertação de Escobar (2011) que a autora não questiona o diagnóstico do TDAH, mas o acata tal como apresentado pelo DSM-IV e procura, por meio da metodologia positivista, encontrar soluções terapêuticas consistentes para o transtorno, sendo bem sucedida em sua empreitada. Posição divergente da tese de Benedetti (2009), defendida no programa de pós-graduação em Educação da UFMS de Campo Grande, em que a autora busca justamente desconstruir o conceito de TDAH e revelar as contradições que lhes são subjacentes. Assim, notamos dois modos muito distintos de abordar cientificamente o TDAH.

\subsubsection{Universidade Federal de Mato Grosso do Sul}

O Programa de pós-graduação em Psicologia da UFMS, nível mestrado teve início em 2011 e apresenta quatro linhas de pesquisa: psicologia e processos psicossociais; psicologia e processos educativos; epistemologia e teoria psicanalítica; e psicologia e processos comportamentais. As temáticas de interesse da linha de pesquisa Psicologia e Processos Psicossociais são: estudos sobre os processos de avaliação psicológica, na perspectiva histórico-cultural; investigações acerca das relações de trabalho e a constituição da subjetividade, conforme o referencial teórico da Teoria Crítica da Sociedade; e pesquisas sobre os aspectos psicossociais do desemprego e da homofobia e as implicações para a consciência e o inconsciente a partir das concepções da Teoria Psicológica Sócio-Histórica. (UFMS, 2014).

Por sua vez, a linha de pesquisa Psicologia e Processos Educativos, segundo o mesmo documento, agrega investigações científicas sobre sujeito e subjetividade em seus processos educativos no referencial da arqueologia do saber; identidade, aprendizagem e constituição do sujeito em espaços educativos; Psicologia e Educação: contornos e possibilidades sob o enfoque das políticas públicas; formas de enfrentamento à violência presente no cotidiano da 
escola no âmbito da Psicologia; constituição identitária nas relações sociais, educativas e de gênero; e relações de gênero e violência. (UFMS, 2014).

Quanto à linha de pesquisa Epistemologia e Teoria Psicanalítica, encontramos o dado de que as pesquisas são orientadas por meio do enfoque dos psicanalistas francófonos Cornelius Castoriadis e Piera Aulagnier. Finalmente, a linha de pesquisa Psicologia e Processos Comportamentais trabalha com o enfoque analítico comportamental na investigação experimental dos determinantes do comportamento verbal explicativo, das propriedades formais e funcionais de regras e na análise de contingências de trechos de instruções nos contextos da saúde, educação e cultura. (UFMS, 2014).

Salientamos que o site não disponibiliza o histórico de implantação do programa, por isso recorremos ao edital do último processo seletivo para descrição das linhas de pesquisa. Conforme a administração do PPGPsi, consultada por meio de correio eletrônico, "o site ainda está em construção" e por isso não apresenta tais informações. A tabela 9, abaixo, apresenta a distribuição das dissertações do PPGPsi segundo os descritores que orientaram as buscas.

Tabela 9. Dissertações PPGPsi UFMS

\begin{tabular}{c|c|c|c}
\hline Ano & N. produções & Medicalização & TDAH \\
\hline 2013 & 04 & 0 & 0 \\
\hline 2014 & 06 & 0 & 0 \\
\hline TOTAL & 10 & 0 & 0 \\
\hline
\end{tabular}

Fonte: Site UFMS. Disponível em: <https://sistemas.ufms.br/sigpos/portal /trabalhos/ buscarPorCurso/cursoId:154> Acesso em: 30 ago. 2014.

O site do PPGPsi da UFMS nos apresenta dados de dois anos, 2013 e 2014, totalizando dez dissertações disponíveis para consulta, destas dez dissertações, duas, defendidas no ano de 2014, não possuem o resumo disponível no link de acesso. Segundo observamos na tabela acima, não consta no programa dissertações que abordam as temáticas da medicalização ou do TDAH.

\section{Considerações Finais}

Finalmente, salientamos que apesar da recente implantação do Estado de Mato Grosso do Sul, que em 2014 completa 37 anos, e dos programas de pós-graduação em Educação e em Psicologia, encontramos expressivo número de trabalhos acadêmicos, totalizando 901 trabalhos. Observamos que os programas de pós-graduação em Educação são os mais antigos, datando de 1988 a implantação do curso de mestrado em Educação na UFMS e de 1994, o 
curso de mestrado em Educação da UCDB. Notamos ainda que no final da primeira década do século XXI há um avanço no número de cursos de mestrado e doutorado, tanto em Educação como em Psicologia, sendo que os programas de pós-graduação em Psicologia são em menor número e mais recentes. Quanto às temáticas em estudo, encontramos um baixo número de produções, denotando que as discussões sobre a medicalização da educação e o TDAH ainda são incipientes no Estado.

Ademais, esta pesquisa permitiu à autora um breve estudo da história do Mato Grosso do Sul e de seus programas de pós-graduação em Educação e em Psicologia, nossa área de interesse. Observamos uma vasta produção acadêmica, apesar da implantação recente dos programas, notamos ainda um aumento no número dos programas de pós-graduação em Educação e em Psicologia no final da última década, mas reiteramos que os programas de pós-graduação em Educação são mais antigos e em maior número e as discussões sobre a medicalização da educação e o TDAH ainda são incipientes no Estado, necessitando de mais investigações. Estes resultados subsidiaram um projeto de pesquisa para o mestrado em Educação em que pretendemos investigar as funções do diagnóstico médico, psicológico e psicopedagógico para professores das séries iniciais do ensino fundamental a partir do viés da medicalização.

Finalmente, acreditamos que o objetivo geral de compreender a abordagem dos temas medicalização e TDAH a partir da análise de teses e dissertações defendidas em programas de Pós-Graduação em Educação e em Psicologia do Estado de Mato Grosso do Sul, e os objetivos específicos deste trabalho - apresentação quantitativa das teses e dissertações, identificação das concepções sobre medicalização e TDAH nas pesquisas realizadas e compreensão das confluências e divergências teóricas e metodológicas identificadas na abordagem dos temas medicalização e TDAH - foi alcançado lançando as bases para futuras investigações sobre as temáticas a possibilitar uma visão crítica da inserção das ciências da saúde na Educação, elucidando os efeitos da medicalização da vida e da Educação.

\section{Referências}

BARBOSA, Eltongil Brandão. História e memória docente nos cursos de letras: Campo Grande, MS. 2012. 163f. Dissertação (Mestrado em Educação) - Universidade Federal da Grande Dourados, Dourados-MS, 2012.

BENEDETTI, Ieda Maria Munhós. Transtorno de déficit de atenção e hiperatividade: os (des)caminhos da construção de um conceito. 2009. 263f. Tese (Doutorado em Educação) Universidade Federal de Mato Grosso do Sul, Campo Grande-MS, 2009. Resumo disponível 
em: <http://www.ppgedu.ufms.br/wp-content/uploads/2014/04/ Resumos-das-Teses2009.pdf $>$ Acesso em 06 ago. 2014.

BITTELBRUNN, Ivonete Bitencourt Antunes. O silêncio da Escola Pública: um estudo sobre os programas de atendimento aos alunos com indicadores de superdotação no Estado de Mato Grosso do Sul. 2003. Dissertação (Mestrado em Educação) - Universidade Federal de Mato Grosso do Sul, Campo Grande-MS, 2003.

COELHO, Helenrose Aparecida da Silva Pedroso. A circulação dos sentidos de promoção da saúde e de prevenção de doenças na mídia impressa. 2007. 235 f. Dissertação (Mestrado em Psicologia) - Universidade Católica Dom Bosco, Campo Grande-MS, 2007.

COLLARES, Cecília Azevedo Lima; MOYSÉS, Maria Aparecida Affonso. A transformação do espaço pedagógico em espaço clínico (a patologização da educação). Série Ideias (23). São Paulo: FDE, 1994. p. 25-31.

Preconceitos no cotidiano escolar: a medicalização do processo ensinoaprendizagem. In: Conselho Regional de Psicologia de São Paulo; Grupo Interinstitucional Queixa Escolar. Medicalização de crianças e adolescentes: conflitos silenciados pela redução de questões sociais a doença de indivíduos. São Paulo, SP: Casa do Psicólogo, 2011. p. 193213.

ESCOBAR, Camila Spengler. As influências da equitação terapêutica no desenvolvimento de crianças e adolescentes com transtorno de déficit de atenção/ hiperatividade. $2011.244 \mathrm{f}$. Dissertação (Mestrado em Psicologia) - Universidade Católica Dom Bosco, Campo GrandeMS, 2011.

OLIVEIRA-JÚNIOR, Mario Balduino de. Representações sociais de saúde e doença de professores de uma universidade pública de Mato Grosso do Sul. 2012. 110 f. Dissertação (Mestrado em Psicologia) - Universidade Católica Dom Bosco, Campo Grande-MS, 2012.

SEVERINO, Antonio Joaquim. O ensino superior brasileiro: novas configurações e velhos desafios. Educar, Curitiba, n. 31, p. 73-89, 2008. Editora UFPR.

SILVA-FILHO, Lourenço Alves da. Educação e política: apontamentos sobre a história da Universidade Estadual de Mato Grosso do Sul (1979-1995). 2008. 175f. Dissertação (Mestrado em História) - Universidade Federal da Grande Dourados, Dourados-MS, 2008.

UNIVERSIDADE ESTADUAL DE MATO GROSSO DO SUL. Programa de pósgraduação em educação: mestrado. Paranaíba, MS, 2013. Disponível em:

<http://www.uems.br/pgedu/arquivos/1_2014-02-25_09-51-05.pdf> Acesso em: 30 jun. 2014. 